Vegane Diät bei Jugendlichen

\section{Wieviel „Grün" ist noch gesund?}

Frage: Ist eine rein vegane Ernährung bei einem 16-jährigen Jugendlichen ohne Bedenken zu vertreten? Sind evtl. bestimmte Nahrungsergänzungsmittel nötig, um z. B. einem Vitamin-B-Mangel vorzubeugen?

Prof. H.S. FüeßI, Haar: Eine streng vegane Ernährung entspricht nicht einer optimalen Ernährung, gerade in einem Alter von 16 Jahren, in dem die körperliche Entwicklung noch nicht voll abgeschlossen ist. Es besteht das Risiko eines Eisen-, Eiweiß- und Vitaminmangels mit Mangelerscheinungen wie Eisen-

mangelanämie und auch Wachstumsstörung. Statt diesem Mangel mit (teuren) Nahrungsergänzungsmitteln, Eisen- und Vitaminpräparaten vorzubeugen, sollte man den jungen Mann auf die Risiken seines Verhaltens hinweisen und auch die Vorteile einer gesunden Mischkost aufzeigen.

Therapie mit Betablockern

\title{
Muss immer die dritte Generation ran?
}

Frage: Bei der Neueinstellung von Patienten auf Betablocker stelle ich mir die Frage, ob ich generell Betablocker der dritten Generation bevorzugen sollte? Von (mehr oder weniger) informierter Patientenseite besteht öfter ein entsprechender Präparatewunsch. Den Hinweis, dass die Präparate der zweiten Generation in meinen Hauptindikationsfeldern (milde Hypertonie junger Patienten, z.T. nervös/stressbedingt, mittelgradige Herzklappeninsuffizienzen, subjektiv störende SVES/ $V E S)$ keinen Nachteil zur dritten Generation aufweisen, teilt man aufgrund des Fortschrittsglaubens nur bedingt. Wie ist ihre Einschätzung?

Prof. Dr. Jürgen C. Frölich, Hannover: Zur Behandlung der arteriellen Hypertonie sollten heute Betablocker der dritten Generation bevorzugt werden. Zusätzlich zur betablockierenden Eigenschaft bewirkt Car- vedilol eine alpha-Blockade; Nebivolol hat einen Nitrat-Effekt. Somit haben diese beiden Wirkstoffe zusätzlich vasodilatierende Eigenschaften und können so zu einer besseren Blutdrucksenkung führen.

Außerdem zeigen sie keine negativen Effekte auf den Glucose- und Lipidstoffwechsel. Bei klinisch stabilen Patienten mit Herzinsuffizienz NYHA II-IV werden neben Carvedilol die Betablocker Bisoprolol und Metoprololsuccinat (kardioselektiv) empfohlen.

Bei COPD-Patienten werden kardioselektive Betablocker bevorzugt. Generell wird eine Betablocker-Therapie mit einer niedrigen Startdosis begonnen, die langsam auftitriert werden kann. Bei Niereninsuffizienz ist je nach Substanz auf eine Dosisanpassung zu achten.

Expertenrat Pharmazie,

www.springermedizin.de

\section{Kinderwunsch bei männlicher Infertilität \\ Spermienqualität natürlich pushen?}

Frage: Ich betreue ein Ehepaar mit Kinderwunsch, der Ehemann leidet unter einer geringen Spermienzahl. Gibt es naturheilkundliche Therapiemöglichkeiten, die Spermienzahl und Aktivität zu steigern, z.B. Phytotherapeutika, Vitamine, Nahrungsergänzungsmittel, Infusionstherapie, etc.?

Dr. Klaus Röttger, Wolfratshausen: Am vielversprechensten ist in der Komplementärmedizin die Akupunktur. Hier gibt es in Studien Hinweise auf einen positiven Effekt. Interessant ist die Feststellung von Siterman et $\mathrm{al}^{1}$, der die Verbesserung der Spermienqualität auf einen Rückgang der skrotalen Hauttemperatur durch Akupunktur zeigen konnte.
Auch in der chinesischen Phytotherapie gibt es Substanzen, wie z.B. Qilin und Qianlie Beixi, die bei Infertilität hilfreich sein können. Weiterhin sind positive Effekte bei Einnahme von Carnitin beschrieben.

Bekanntermaßen führt auch Adipositas zu einer veringerten Fertilität ${ }^{2}$. Bei eventuellem Übergewicht wäre eine Lebensstiländerung hier durchaus lohnend.

' Siterman S et al, Success of acupuncture treatment in patients with initially low sperm output is associated with a decrease in scrotal skin temperature, Asian J

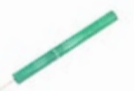

Nadeln bringen die Manneskraft wieder in Schwung.

Androl 2009, 11:200-208

${ }^{2} \mathrm{Hammoud} \mathrm{AO}$ et al, Obesity and male infertility: a practical approach. Seminars in reproductive medicine 2012, 30: 486-495.

Expertenrat Naturheilverfahren, wWw.springermedizin.de 